\title{
Examination of Schrodinger Equation in Pre-Planckian Space-Time Early Universe
}

\author{
Andrew Walcott Beckwith \\ Physics Department, College of Physics, Chongqing University Huxi Campus, Chongqing, China \\ Email: Rwill9955b@gmail.com, abeckwith@uh.edu
}

How to cite this paper: Beckwith, A.W. (2017) Examination of Schrodinger Equation in Pre-Planckian Space-Time Early Universe. Journal of High Energy Physics, Gravitation and Cosmology, 3, 21-28. http://dx.doi.org/10.4236/jhepgc.2017.31004

Received: August 29, 2016

Accepted: November 20, 2016

Published: November 23, 2016

Copyright $\odot 2017$ by author and Scientific Research Publishing Inc. This work is licensed under the Creative Commons Attribution International License (CC BY 4.0).

http://creativecommons.org/licenses/by/4.0/

\begin{abstract}
We look at the Vuilli (1999) write up of a generalized Schrodinger equation with its Ricci scalar inclusion, in curved space-time. This has a simplified version in Pre-Planckian regime, which leads to comparing a resultant admissible wave function with Bohmian reformulations of quantum physics. As was done earlier, we compare this result with a formulation of a modified "Poisson" equation from Poissons and Will from 2014, and then use inflaton physics. The resulting inflaton is then compared to the wave functional in the first part of this document.
\end{abstract}

\section{Keywords}

Ricci Tensor, Schrodinger Equation, Modified Poisson Equation,

Massive Gravity, Inflaton Physics

\section{Vuilli Treatment of Schrodinger Equation for Curved Space-Time}

Here, we bring up [1], and a reset of the Schrodinger equation in early space-time, with curvature.

In [1] we start with

$$
i \hbar \partial_{t} \Psi=-\frac{\hbar^{2}}{2 m} \nabla^{2} \Psi+m c^{2} \Psi
$$

Through the following substitutions,

$$
\begin{aligned}
& \nabla^{2} \rightarrow\left(\frac{p^{\alpha} p^{\beta}}{m^{2} c^{2}}-g_{\alpha \beta}\right) \nabla_{\alpha} \nabla_{\beta} \\
& \& p^{\alpha}=i \hbar \cdot g^{\alpha \beta} \nabla_{\beta} \\
& p_{\beta}=i \hbar \cdot \nabla_{\beta} \\
& \& m c^{2}=\frac{g^{\alpha \beta} p_{\alpha} p_{\beta}}{m}
\end{aligned}
$$


Then, after more derivation [1] obtained, with using the Ricci tensor $R_{a}^{c}$ the following result: [2]

$$
\nabla^{a} \nabla_{a}\left(\nabla^{b} \nabla_{b}+\frac{m^{2} c^{2}}{\hbar^{2}}\right) \Psi+\nabla^{a} R_{a}^{c} \nabla_{c} \Psi=0
$$

\section{Simplifying Equation (3) in Pre-Planckian Space-Time}

We will re write Equation (3) to read as follows, with the result that in Pre-Planckian space-time

$$
\left(\partial_{r}^{4}+\frac{m^{2} c^{2}}{\hbar^{2}} \partial_{r}^{2}\right) \Psi+R_{r}^{r} \partial_{r}^{2} \Psi=0
$$

Ricci tensor $R_{a}^{c}$ in this setting becomes a constant, and is part of how the wave function evolves, and our candidate wave functional takes the form of

$$
\Psi \propto \Psi_{\text {initial }} \mathrm{e}^{\aleph r} \sim \Psi_{\text {initial }} \exp \left(i \sqrt{\frac{m^{2} c^{2}}{\hbar^{2}}+R_{r}^{r}} \cdot r\right)
$$

We will be identifying what to put into [3], i.e., the Ricci scalar as given in PrePlanckian space-time

$$
\begin{aligned}
R_{a}^{c} \underset{\text { Pre-Planckian }}{\longrightarrow} R_{r}^{r} & =6 \cdot\left(\left[\frac{\ddot{a}}{a}\right]+\left[\frac{\dot{a}}{a}\right]^{2}+\frac{k \text { (flatness })}{a^{2}}\right) \\
& =12 \cdot H_{\text {initial }}^{2}+\frac{6 k(\text { flatness })}{a_{\text {initial }}^{2}} \sim 12 \cdot H_{\text {initial }}^{2}
\end{aligned}
$$

We are assuming that the term $k$ (flatness) approaches zero in Pre-Planckian spacetime. Obviously if it did not, the last term $\frac{6 k \text { (flatness) }}{a_{\text {intitial }}^{2}}$ would be dominant.

Having said this, with an evolutionary equation statement as to the phase value of $\Psi$, it is time to look at the initial $\Psi_{\text {initial }}$ and to try to learn some physics from it.

\section{Defining the Initial Value $\Psi_{\text {initial }}$ of Equation (5)}

In order to do this, it may be useful to look at the classical degeneracy argument for forming $\Psi_{\text {initial }}$, and the reference by Rubakov may be useful for this [4] i.e. using a false vacuum analogy, we can write, if $q$ is a generalized space-time unit of "length", and we examine a quartic potential, i.e. look at

$$
\begin{aligned}
& V(q)=-\frac{\mu^{2} \cdot q^{2}}{2}+\frac{\tilde{\lambda} \cdot q^{4}}{4} \\
& \& S(\text { action })=\int_{q_{0}^{-}}^{q_{0}^{+}} \sqrt{2 M V(q)} \mathrm{d} q \\
& \Rightarrow \Psi_{\text {initial }} \propto \Psi_{0}^{+} \sim \exp \left(-\int_{0}^{q^{+}} \mathrm{d} q \sqrt{2 M V(q)}\right)
\end{aligned}
$$

Now, use the usual given 


$$
\begin{aligned}
& \frac{M}{2} \frac{\mathrm{d} q}{\mathrm{~d} \tau}=\frac{M c}{2}=V(q) \\
& \Rightarrow \Psi_{\text {initial }} \propto \Psi_{0}^{+} \sim \exp \left(-\int_{0}^{q^{+}} \mathrm{d} q M \sqrt{c}\right) \\
& \underset{c=1}{\longrightarrow} \Psi_{0}^{+} \sim \exp \left(-M q^{+}\right)
\end{aligned}
$$

If using $\mathrm{Ng}$ infinite quantum statistics, [5], and a non zero massive graviton mass (massive gravity) [6]

$$
E=M=[S(\text { entropy }) \sim n(\text { count-gravitons })] \cdot m_{\text {graviton }}
$$

And if we have here that $q^{+}$is in some sense proportional to length less than or equal to Planck length, the astonishing conclusion is that Equation (8) would probably be biased toward a low ( nonzero) entropy count, which would mean for finite initial entropy, connected with information transfer, that we would through Equation (8) and Equation (9) have a bias toward initially low, say $10^{5}$ or so initial entropy, as a way to quantify the input into the formation of an initial wavefunction, using entropy as equivalent to information as given by Lloyd [7].

\section{Comparing the Inputs into Equation (5), Equation (6), Equation (8) and Equation (9) against Force against Individual "Gravitons" in the Pre-Planckian Space-Time}

We will go to the [3] reference, page 85 , in order to look at a change in the stress energy tensor,

$$
\begin{aligned}
& \Delta T^{00}=\left(-T_{, j}^{0 j}+F^{(0)}\right) \cdot \Delta t \\
& F^{(0)}=\text { force/fluid (unit-volume) }
\end{aligned}
$$

Using this, and stating that in the Pre-Planckian regime of space-time due to [8], that $T_{, j}^{0 j} \approx 0$, then if so, using [3] and [8]

$$
\begin{aligned}
& F^{(0)}=\text { force/fluid (unit-volume) } \\
& T_{, j}^{0 j} \approx 0 \\
& \Rightarrow \Delta T^{00}=\left(F^{(0)}\right) \cdot \Delta t \\
& \Delta E=\left(F^{(0)}\right) \cdot \Delta V^{(4)}=\frac{1}{\Delta t \cdot a_{\text {initial }}^{2} \phi} \\
& \Rightarrow F^{(0)}=\frac{1}{\Delta V^{(3)}(\Delta t)^{2} \cdot a_{\text {initial }}^{2} \phi} \\
& =\text { force/fluid (unit-volume) }
\end{aligned}
$$

Here, we have that the initial volume $\Delta V^{(3)}$ would be less than the cube power of a Planck length [9], but not zero, whereas the initial time would be less than, Planck time, but not equal to zero, [9]. For our analysis in the Pre-Planckian regime, we will specify $a_{\text {intial }}^{2}$ as the square of a nonzero initial scale factor for a nonsingular regime of space-time for General relativity with a value as given by [10], and elaborated upon in [11]. In addition we have that the inflaton, as given in Equation (11) is explained in context by [12], and we will use an argument below as to how a nonzero graviton mass 
is linkable to a non zero initial radii, which in turn will state that it is highly unlikely that $\Delta V^{(3)}$ which is proportional to a radial distance, cubed, goes to zero.. Note that Equation (11) is also an argument as to why there would be a finite, not almost "infinite" initial value of entropy, so then that Equation (8) would not go to zero.

Having said that, let us use a semi classical argument as to why the radii would not go to zero, even in Pre-Planckian space-time. This would be to insure that $\Delta V^{(3)}$ would not go to zero, even in Pre-Planckian space-time

\section{What Is Important about the Modified Poissons Equation [12]? Getting a Non Zero Initial $\Delta V^{(3)}$}

We will first of all refer to two necessary and sufficient conditions for the onset of a massive graviton given in [13], and combined with Padmanablan's reference [12].

i.e. what we will be doing is to re do the reference calculations given in [13] with

$$
\left(\nabla^{2}+\left[\lambda^{-2}=\left(\frac{m_{\text {graviton }} c}{\hbar}\right)^{2}\right]\right)\left[U=\frac{G m}{r} \cdot \exp \left[(-r / \lambda)=\left(\frac{r \cdot m_{\text {graviton }} c}{\hbar}\right)\right]\right]=-4 \pi G \rho
$$

Here, we will be using in the Pre-Planckian potential the inputs from the data usually associated with [12]

$$
\begin{aligned}
& a \approx a_{\min } t^{\gamma} \\
& \Leftrightarrow \phi \approx \sqrt{\frac{\gamma}{4 \pi G}} \cdot \ln \left\{\sqrt{\frac{8 \pi G V_{0}}{\gamma \cdot(3 \gamma-1)}} \cdot t\right\} \\
& \Leftrightarrow V \approx V_{0} \cdot \exp \left\{-\sqrt{\frac{16 \pi G}{\gamma}} \cdot \phi(t)\right\}
\end{aligned}
$$

In other words, we will be using the inflation given by

$$
\phi \approx \sqrt{\frac{\gamma}{4 \pi G}} \cdot \ln \left\{\sqrt{\frac{8 \pi G V_{0}}{\gamma \cdot(3 \gamma-1)}} \cdot t\right\}
$$

If so, then

$$
\left[\frac{\mathrm{d}^{2}}{\mathrm{~d} r^{2}}+\left(\frac{m_{\text {graviton }} c}{\hbar}\right)^{2}\right] \cdot\left(\frac{r^{-1} \alpha \cdot(3 \alpha-1)}{32 \pi^{2}}\right)=G \cdot \rho
$$

Then, after algebra, we have the following, from [14]

$$
\left(m_{\text {graviton }}\right)^{2} \approx\left[\frac{32 \pi^{2} r \cdot \hbar^{2} \cdot G \cdot \rho}{c^{2} \cdot \alpha \cdot(3 \alpha-1)}-\frac{16 \pi^{2} r^{-1} \cdot \hbar^{2} \cdot G^{-2}}{c^{2} \cdot \alpha \cdot(3 \alpha-1)}\right]
$$

The quadratic Equation this engenders is, how to say

$$
\begin{aligned}
& \left(m_{\text {graviton }}\right)^{2} \approx\left[\frac{32 \pi^{2} r \cdot \hbar^{2} \cdot G \cdot \rho}{c^{2} \cdot \alpha \cdot(3 \alpha-1)}-\frac{16 \pi^{2} r^{-1} \cdot \hbar^{2} \cdot G}{c^{2} \cdot \alpha \cdot(3 \alpha-1)}\right] \\
& \Rightarrow r^{2}-\frac{r \cdot\left(m_{\text {graviton }}\right)^{2}}{\left(\frac{32 \pi^{2} \hbar^{2} \cdot G \cdot \rho}{c^{2} \cdot \alpha \cdot(3 \alpha-1)}\right)}-\frac{\left(\frac{16 \pi^{2} \cdot \hbar^{2} \cdot G}{c^{2} \cdot \alpha \cdot(3 \alpha-1)}\right)}{\left(\frac{32 \pi^{2} \hbar^{2} \cdot G \cdot \rho}{c^{2} \cdot \alpha \cdot(3 \alpha-1)}\right)} \\
& \doteq r^{2}-\frac{r \cdot\left(m_{\text {graviton }}\right)^{2}}{\left(\frac{32 \pi^{2} \hbar^{2} \cdot G \cdot \rho}{c^{2} \cdot \alpha \cdot(3 \alpha-1)}\right)}-\frac{1}{2 \rho}=0
\end{aligned}
$$


A candidate for the density functional will come next, with the way of obtaining a critical value for $r$ is given by [14] as follows, i.e. if

$$
V_{\text {Pre-Planckian }} \sim\left(\Delta E \sim \frac{\hbar}{\delta t \cdot a_{\text {min }}^{2} \phi_{\text {inf }}}\right)
$$

As far as applications to: [1]

$$
\begin{aligned}
& r^{2}-\frac{r^{4} \cdot\left(m_{\text {graviton }}\right)^{2} a_{\text {min }}^{2} \cdot \delta t \cdot \sqrt{\frac{\gamma}{4 \pi G} \cdot \ln \left\{\sqrt{\frac{8 \pi G V_{0}}{\gamma \cdot(3 \gamma-1)}} \cdot t\right\}}}{\left(\frac{32 \pi^{2} \hbar^{2} \cdot G}{c^{2} \cdot \alpha \cdot(3 \alpha-1)}\right)} \\
& -\frac{r^{3} a_{\min }^{2} \delta t \cdot \sqrt{\frac{\gamma}{4 \pi G}} \cdot \ln \left\{\sqrt{\frac{8 \pi G V_{0}}{\gamma \cdot(3 \gamma-1)}} \cdot t\right\}}{2}=0
\end{aligned}
$$

This would, lend itself to a quadratic equation for $r$, and the cube of $r$ would be proportional to $\Delta V^{(3)}$ which would be non zero, in Pre-Planckian space-time conditions.

\section{Comparing the Results of a Non Zero $\Delta V^{(3)}$ in \\ Pre-Planckian Wave Functional for Equation (8) against \\ De Broglie-Bohmian Path of a Wave Functional}

What we are examining if our qualitative argument which in sum yields a PrePlanckian wavefunction as compared against the construction given in [15] which is in spirit comparable, up to a point with [16]

According to [15] we would have

$$
\Psi(x, t)=\Psi\left(x_{0}, t_{0}\right) \exp \left[\left(\frac{i}{\hbar} \int_{x_{0}, t_{0}}^{x, t} \mathrm{~d} t\left[\frac{(\nabla S(\text { action }))^{2}}{2 m}-(Q+V)\right]\right)-\int_{x_{0}, t_{0}}^{x, t} \mathrm{~d} t \frac{\left(\nabla^{2} S(\text { action })\right)}{2 m}\right]
$$

Here, $S$ (action) would be the same as Equation (7), whereas we have $V$ as given by Equation (18), and then we have

$$
\begin{aligned}
& R=\operatorname{Re} \Psi(x, t) \\
& Q=\frac{-\hbar^{2} \nabla^{2} R}{2 m R} \\
& \nabla \underset{\text { Pre-Planckian }}{\stackrel{2}{\longrightarrow}} \partial_{r}
\end{aligned}
$$

Here, if we use $\hbar=c=1$, and have $\Psi\left(x_{0}, t_{0}\right)$ as given by Equation (8) whereas we find

$$
\begin{aligned}
& \hbar=c=1 \\
& Q=\frac{-\hbar^{2} \nabla^{2} R}{2 m R} \underset{\text { Pre-Planckian }}{\longrightarrow} \frac{1}{2 M} \cdot\left(m_{g}^{2}+R_{r}^{r}\right)
\end{aligned}
$$

Here, $M$ would be given by Equation (9), i.e. and $V$ were given by Equation (18) we would find to a point that

$$
\Psi(x, t) \sim \Psi\left(x_{0}, t_{0}\right) \exp \left[\left(\frac{i}{\hbar} \int_{x_{0}, t_{0}}^{x, t} \mathrm{~d} t\left[\frac{(\nabla S(\text { action }))^{2}}{2 m}-(Q+V)\right]\right)\right]
$$


i.e. the term $\Psi\left(x_{0}, t_{0}\right)$ would likely be the same, but interesting enough, $\int_{x_{0}, t_{0}}^{x_{0} t} \mathrm{~d} t \frac{\left(\nabla^{2} S(\text { action })\right)}{2 m}$ would likely be almost zero, i.e. not contributing at all.

\section{Conclusion, Overlap with the Bohmian Quantum Picture of Physics If Equation (24) Is Confirmed for Pre-Planckian Space-Time}

The question to ask, is the following true? This has to be confirmed rigorously.

$$
\int_{x_{0}, t_{0}}^{x, t} \mathrm{~d} t \frac{\left(\nabla^{2} S(\text { action })\right)}{2 m} \underset{\text { Pre-Planckian }}{\longrightarrow} \varepsilon^{+} \sim 0
$$

The significance of proving or falsifying Equation (24) will in the end be part of future data analysis which should not contravene [17] [18] [19]. i.e. the experimentally implied limits should be adhered to and studied rigorously.

Furthermore, we have that analysis of Equation (24) may be in tandem with analysis of the Corda paper [20] as to if gravity is possibly scalar-tensor, an extension of GR (possibly with some semi classical treatment of presumed quantum gravity formulations), or something else.

Finally, since our paper is with respect to relic conditions, if so, then if we are to use a variant of interferometer methods, reference [21] and [22] if relic conditions are observable, via some form of space bound system, may allow us to with refinements get enough control of stochastic noise contamination of GW and the foot print of massive gravity to come up with confirmable data sets as to early universe conditions. With luck, with considerable refinement of instrumentation, we may also be able to get experimental confirmation of [23] and its predictions as to inflaton physics and possibly massive gravity.

\section{Acknowledgements}

This work is supported in part by National Nature Science Foundation of China grant No. 11375279.

\section{References}

[1] Vuille, C. (1999) Schrodingers' Equation in General Relativity. In: Burgess, C.P. and Myers, R., Eds., General Relativity and Relativistic Astrophysics, Eighth Canadian Conference, AIP Conference Proceedings, 493, Melville, New York, 243-246.

[2] Carmelli, M. (1982) Classical Fields, General Relativity and Gauge Theory. Wiley Interscience, New York.

[3] Scott, R. (2016) A Student Manual for A First Course in General Relativity. Cambridge University Press, Cambridge, UK.

[4] Rubakov, V. (2002) Classical Theory of Gauge Fields. Princeton University Press, Princeton.

[5] Ng, Y.J. (2008) Spacetime Foam: From Entropy and Holography to Infinite Statistics and Nonlocality. Entropy, 10, 441-461. https:/doi.org/10.3390/e10040441

[6] Goldhaber, A. and Nieto, M. (2010) Photon and Graviton Mass Limits. Reviews of Modern 
Physics, 82, 939-979. http://arxiv.org/abs/0809.1003 https:/doi.org/10.1103/RevModPhys.82.939

[7] Lloyd, S. A Theory of Quantum Gravity Based on Quantum Computation. https://arxiv.org/abs/quant-ph/0501135

[8] Beckwith, A. (2016) Gedanken Experiment for Refining the Unruh Metric Tensor Uncertainty Principle via Schwarzschild Geometry and Planckian Space-Time with Initial Nonzero Entropy and Applying the Riemannian-Penrose Inequality and Initial Kinetic Energy for a Lower Bound to Graviton Mass (Massive Gravity). Journal of High Energy Physics, Gravitation and Cosmology, 2, 106-124. https:/doi.org/10.4236/jhepgc.2016.21012

[9] Schwartz, B. (2011) A First Course in General Relativity. 2nd Edition, Cambridge University Press, Cambridge, United Kingdom.

[10] Camara, C.S., de Garcia Maia, M.R., Carvalho, J.C. and Lima, J.A.S. (2004) Nonsingular FRW Cosmology and Non Linear Dynamics. Version 1, February 12. Arxiv astro-ph/0402311.

[11] Beckwith, A. (2016) Thermodynamics and the Energy of Gravity Waves, GW's, as a Consequence of Non Linear Electrodynamics, NLED, Leading to an Initial Temperature and Strain h for Gravity Waves, GW, at the Start of Inflation. Journal of High Energy Physics, Gravitation and Cosmology, 2, 98-105. https:/doi.org/10.4236/jhepgc.2016.21011

[12] Padmanabhan, T. (2005) Understanding Our Universe, Current Status and Open Issues. In: Ashatekar, A., Ed., 100 Years of Relativity, Space-Time Structure: Einstein and Beyond, World Scientific Publishing, Singapore, 175-204. http://arxiv.org/abs/gr-qc/0503107 https:/doi.org/10.1142/9789812700988_0007

[13] Poisson, E. and Will, C. (2014) Gravity: Newtonian, Post Newtoninan, Relativistic. Cambridge University Press, Cambridge. https:/doi.org/10.1017/CBO9781139507486

[14] Beckwith, A. (2016) Examination of Sufficient Conditions for Forming Mass of "Massive Graviton", from Early Universe.

[15] Licata, I. and Fiscaletti, D. (2014) Quantum Potential: Physics, Geometry, and Algebra. Springer-Verlag, New York. https:/doi.org/10.1007/978-3-319-00333-7

[16] Beckwith, A. (2016) Gedanken Experiment for Looking at $\delta \mathrm{g}_{t t}$ for Initial Expansion of the Universe and Influence on HUP via Dynamical Systems, with Positive Pre-Planckian Acceleration. Journal of High Energy Physics, Gravitation and Cosmology, 2, 531-545. https:/doi.org/10.4236/jhepgc.2016.24046

[17] Abbott, B., et al. (2016) Observation of Gravitational Waves from a Binary Black Hole Merger. LIGO Scientific Collaboration and Virgo Collaboration. Physical Review Letters, 116, Article ID: 061102. https:/doi.org/10.1103/physrevlett.116.061102

[18] Abbott, B., et al. (2016) GW151226: Observation of Gravitational Waves from a 22-SolarMass Binary Black Hole Coalescence. LIGO Scientific Collaboration and Virgo Collaboration. Physical Review Letters, 116, Article ID: 241103. https:/doi.org/10.1103/physrevlett.116.241103

[19] Abbott, B., et al. (2016) Tests of General Relativity with GW150914. Physical Review Letters, 116, Article ID: 221101. https://arxiv.org/pdf/1602.03841.pdf https:/doi.org/10.1103/physrevlett.116.221101

[20] Corda, C. (2009) Interferometric Detection of Gravitational Waves: The Definitive Test for General Relativity. International Journal of Modern Physics D, 18, 2275-2282.

https://arxiv.org/abs/0905.2502 https:/doi.org/10.1142/S0218271809015904

[21] Willke, B., et al. (2006) Stabilized High Power Laser for Advanced Gravitational Wave Detectors. Journal of Physics. Conference Series, 32, 270.

http://iopscience.iop.org/article/10.1088/1742-6596/32/1/040/meta;jsessionid=B072B9D72E 
7EB4F993D70061EEE365CF.c5.iopscience.cld.iop.org

https:/doi.org/10.1088/1742-6596/32/1/040

[22] Flaminio, R., Franc, J., Michel, C., Morgado, N., Pinard, L. and Sassolas, B. (2010) A Study of Coating Mechanical and Optical Losses in View of Reducing Mirror Thermal Noise in Gravitational Wave Detectors. Classical and Quantum Gravity, 27, Article ID: 084030. https:/doi.org/10.1088/0264-9381/27/8/084030

[23] Corda, C. (2012) Gravity's Primordial Breath. Electronic Journal of Theoretical Physics, 9 , 1-10. http://www.ejtp.com/articles/ejtpv9i26p1.pdf

Submit or recommend next manuscript to SCIRP and we will provide best service for you:

Accepting pre-submission inquiries through Email, Facebook, LinkedIn, Twitter, etc. A wide selection of journals (inclusive of 9 subjects, more than 200 journals)

Providing 24-hour high-quality service

User-friendly online submission system

Fair and swift peer-review system

Efficient typesetting and proofreading procedure

Display of the result of downloads and visits, as well as the number of cited articles

Maximum dissemination of your research work

Submit your manuscript at: http://papersubmission.scirp.org/

Or contact jhepgc@scirp.org 